Reprod. Nutr. Dévelop., 1981, 21 (5A), 621-627.

\title{
Liaison spécifique du cortisol aux protéines plasmatiques chez le Cobaye, de la naissance au sevrage
}

\author{
par J. M. SCHNEIDER de ZAJOL, P. DELOST \\ Laboratoire de Physiologie Animale et E.R.A. C.N.R.S. d'Endocrinologie du Développement, \\ Université de Clermont-Ferrand, Ensemble Scientifique des Cézeoux, B.P. 45, 63170 Aubière, France.
}

Summary. Specific binding of cortisol to plasma protein in the guinea-pig from birth to weaning.

We have estimated two parameters of guinea-pig adrenal function during the neonatal period :

- the concentration of plasma cortisol by competitive binding to protein ;

- the association constant, $\mathrm{Ka}$, and total capacity of CBG binding (NS) by the thin-layer gel filtration technique.

The concentration of plasma cortisol, very high at birth, declined markedly between neonatal days 1 and 10 and then remained constant between days 10 and 20, the time of weaning.

$\mathrm{Ka}$ was $0.25 .10^{7} . \mathrm{M}^{-1}$ in the newborn and the adult of both sexes. This value was lower than that of other species. NS, which was very high at birth $(111 \pm 5 \mu \mathrm{g} / 100 \mathrm{ml}$ in the male ; $101 \pm 5 \mu \mathrm{g} / 100 \mathrm{ml}$ in the female), decreased rapidly during the first two neonatal days.

At that time, it reached adulf levels in the female $(45 \pm 5 \mu \mathrm{g} / 100 \mathrm{ml})$ and remained stable. Adult binding capacity $(16 \pm 2 \mu \mathrm{g} / 100 \mathrm{ml})$ was reached in the male after neonatal day 20.

\section{Introduction.}

Afin de mieux connaître l'activité corticosurrénalienne au cours des premiers jours de la vie chez le Cobaye el de compléter les informations déjà acquises en période néonatale sur la cortisolémie, le cortisol intra-surrénalien (Dalle et Delost, 1974) ef le taux de clearance métabolique (Dalle et Delost, 1979), nous avons entrepris l'étude de la liaison spécifique du cortisol aux protéines plasmatiques chez le jeune Cobaye de la naissance $(0 \mathrm{~h})$ au sevrage (20e jour). Les résultats obtenus permettent de préciser la qualité et la quantité des activités liantes CBG au cours d'une période physiologique caractérisée par l'adaptation de la surrénale à des conditions d'existence autonome. Leur comparaison avec la cortisolémie à chacun des stades post-nataux étudiés permet également d'estimer l'évolution de la fraction libre de l'hormone responsable de l'activité biologique. 


\section{Matériel ef méthodes.}

Animaux ef préparation des plasmas. - Les expériences sonł réalisées sur des cobayes de souche Dunkin-Hartley élevés dans des conditions identiques de température $\left(20 \pm 2^{\circ} \mathrm{C}\right)$ et d'éclairement (photopériodisme naturel).

Les lois d'animaux ( $n=8-12$ par sexe), correspondant aux stades 7 jours, 10 jours, 20 jours et 180 jours ont été sacrifiés par décapitation entre $9 \mathrm{~h}$ et $11 \mathrm{~h}$; les lots $0 \mathrm{~h}$ et $50 \mathrm{~h}$, aux temps exacts correspondants, en prenant l'heure de naissance pour origine.

Le sang artério-veineux est recueilli sur héparine ; le plasma séparé par centrifugation est congelé à $-30^{\circ} \mathrm{C}$ et ne subit qu'une seule décongélation au moment du dosage.

Dosage de la cortisolémie. - La cortisolémie est mesurée sur chaque animal suivant la technique de liaison compétitive aux protéines (Murphy, 1967). Les hormones sont extraites par le chloroforme et séparées par chromatographie sur papier dans le système Bush no 5 (1952). Elles sont éluées par le méthanol ef divisées en deux fractions. L'une est utilisée pour déterminer le pourcentage de récupération de $4{ }^{14} \mathrm{C}$-cortisol (New England Nuclear corporation) ajouté aux échantillons plasmatiques qui est trouvé égal à 80 p. 100 . Le cortisol de la seconde fraction est dosé par compétition contre une solution de protéine liante, correspondant à un plasma de chien exempt de cortisol endogène, dilué à 2,5 p. 100 et équilibré avec du $1-2{ }^{3} \mathrm{H}$ cortisol (New England Nuclear (Co). La sensibilité de la méthode est de $0,5 \mathrm{ng}$ pour le cortisol, sa précision égale à 10 p. 100 entre 2 et 15 ng d'hormone.

Détermination des paramètres de liaison du cortisol à la CBG. - Considérant le faible volume d'échantillon plasmatique à notre disposition, nous avons utilisé une microtechnique de filtration sur gel de Sephadex en couche mince avec membrane de Cuprophan (Technicon LTD) comme support, adaptée de celle proposée par Topert et al. (1974). Cette filtration est conduite avec l'appareil TLG fourni par Pharmacia Fine Chemical.

Le gel est étalé en couche de 0,8 mm d'épaisseur ; il est de qualité G. 75 SF gonflé dans un tampon phosphate $0,015 \mathrm{M} \mathrm{pH}=7,40$ qui sert également de phase mobile.

Les échantillons plasmatiques non dilués de $10 \mu$ l déposés sur la plaque sont développés dans les conditions suivantes :

Angle d'inclinaison de la plaque : $30^{\circ}$; température : $+4^{\circ} \mathrm{C}$; distance de migration : $7,5 \mathrm{~cm}$ repérée par utilisation de Ferritine (Sigma Chemical Cie) servant d'indicateur de migration.

Après développement, la membrane cuprophan supportant le gel est découpée transversalement en bandes de $4 \mathrm{~mm}$ de largeur, placées dans des fioles de comptage et additionnées de $10 \mathrm{ml}$ de liquide scintillant, de composition suivante : Dioxane : $1 \mathrm{I}$; Naphtalène : $20 \mathrm{~g}$; PPO : $4 \mathrm{~g}$; POPOP : 0,1 g. Le comptage de la radioactivité est effectué avec un scintillateur Packard.

Les mesures des fractions libres ef liées de l'hormone pour la détermination de Ka (constante d'association du cortisol à la CBG) ont été faites sur des pool de plasma de chaque stade préalablement débarrassé du cortisol endogène et incubé avec une dose fixe $(250000 \mathrm{dpm})$ de $1-2{ }^{3} \mathrm{H}$ cortisol (Act. Spe. $=80-100 \mathrm{Ci} / \mathrm{mmol} \mathrm{NEN}$ ) et des quan- 
tités croissantes de cortisol non marqué. Pour le calcul de $\mathrm{Ka}$, nous avons utilisé la méthode graphique de Scatchard (1949), la pente de la droite de régression correspondante fournit la valeur de $\mathrm{Ka}$; (NS) qui est la capacité totale de liaison de la protéine spécifique est déterminée sur les plasmas individuels, constitutifs de chaque lot, incubés avec une dose de cortisol tritié égale à $250000 \mathrm{dpm}$ plus une quantité fixe de cortisol non marqué correspondant à la saturation de la liaison spécifique. Dans ces conditions (NS) peut être calculé à partir de la radioactivité liée à la protéine.

\section{Résultats.}

Evolution de la cortisolémie entre la naissance ef le vingtième jour chez le Cobaye des deux sexes. - Les concentrations plasmatiques en cortisol et leur évolution en période post-natale chez le mâle et la femelle sont présentées sur les figures 1 et 2.

Constante d'association Ka du cortisol à la CBG chez le Cobaye mâle ef femelle en période néonatale (tabl. 1). - Le tracé, en représentation de Scatchard, des droites de régression obtenues à partir des valeurs expérimentales (5 déterminations sur pool de plasma par stade et par sexe) permet de déterminer Ka, égal à la pente de la droite obtenue.

\section{TABLEAU 1}

Constante d'association $\mathrm{Ka}\left(\mathrm{M}^{-1}\right)$ à $4^{\circ} \mathrm{C}$ du cortisol d̀ la $\mathrm{CBG}$ chez le Cobaye des deux sexes au cours de la période néonatale

\begin{tabular}{|c|c|c|c|c|c|c|}
\hline \multirow[b]{2}{*}{ Sexe } & \multicolumn{6}{|c|}{ Age } \\
\hline & $\mathrm{Oh}$ & $50 \mathrm{~h}$ & 7 jours & 10 jours & 20 jours & $\begin{array}{l}\text { Témoins } \\
180 \text { jours }\end{array}$ \\
\hline $\begin{array}{l}\text { Mâle } \ldots . . . \\
\text { Femelle ... }\end{array}$ & $\begin{array}{l}0,26 \cdot 10^{7} \\
0,26 \cdot 10^{7}\end{array}$ & $\begin{array}{l}0,29 \cdot 10^{7} \\
0,30 \cdot 10^{7}\end{array}$ & $\begin{array}{l}0,27 \cdot 10^{7} \\
0,23 \cdot 10^{7}\end{array}$ & $\begin{array}{l}0,30 \cdot 10^{7} \\
0,21 \cdot 10^{7}\end{array}$ & $\begin{array}{l}0,16 \cdot 10^{7} \\
0,18 \cdot 10^{7}\end{array}$ & $\begin{array}{l}0,29 \cdot 10^{7} \\
0,24 \cdot 10^{7}\end{array}$ \\
\hline
\end{tabular}

Evolution de la capacité totale de liaison spécifique (NS) au cours du développement néonatal chez le Cobaye des deux sexes (fig. 1 et 2). - Ce second paramètre, caractéristique d'un système de liaison spécifique, est, contrairement au précédent, très influencé par les conditions physiologiques (sexe, âge de l'animal) ainsi que par l'état d'activité des autres glandes endocrines (Gala et Westphal, 1966).

Les résultats montrent qu'à la naissance, les concentrations de cortisol fixées spécifiquement (exprimées en $\mu \mathrm{g} / 100 \mathrm{ml}$ de plasma) sont élevées aussi bien chez le mâle $(112,5 \pm 5,0)$ que chez la femelle $(101,0 \pm 5,0)$; elles diminuent rapidement au cours des deux premiers jours de la vie.

Chez la femelle, dès la $50^{e}$ heure, la capacité moyenne de l'adulte $(44,9 \pm 5,1 \mu \mathrm{g} /$ $100 \mathrm{ml}$ ) est atteinte et se stabilise à ce niveau. Chez le mâle, la décroissance de (NS) se prolonge de manière ralentie encore aprèz le $20^{e}$ jour post-natal ; les valeurs de l'adulte $(16,1 \pm 1,7 \mu \mathrm{g} / 100 \mathrm{ml})$ ne sont retrouvées qu'après ce stade.

La comparaison de (NS), chez le mâle et la femelle, ne montre aucune différence significative lors des deux premiers stades $(0 \mathrm{~h}$ et $50 \mathrm{~h} ; 0,1<P<0,2)$. Dès le $7 \mathrm{e}$ jour, 

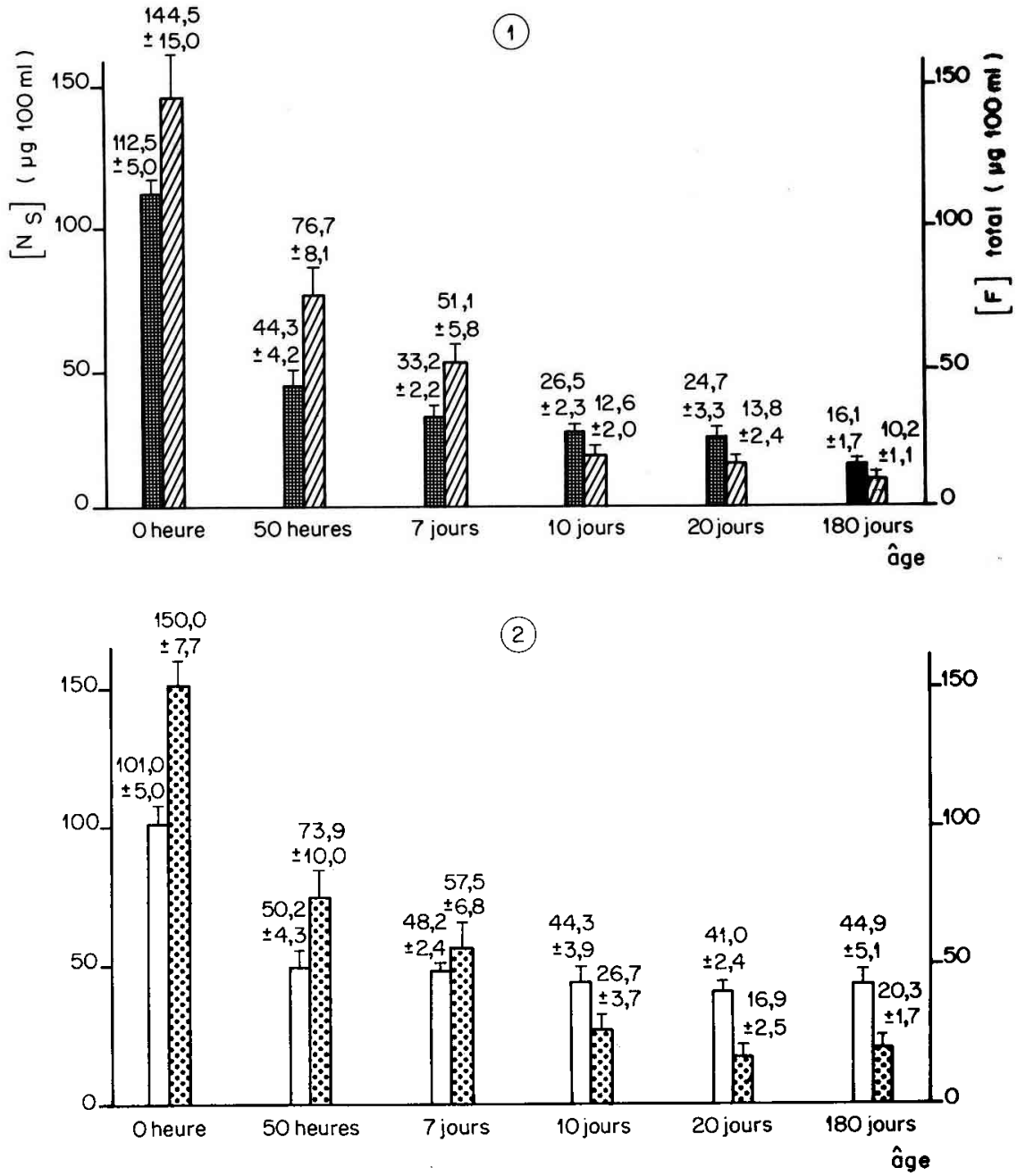
(1) môle $\left\{\begin{array}{l}\text { cortisolémie } \\ \text { copacité totale de }\end{array}\right.$ lioison
(2) femelle $\left\{\begin{array}{l}\text { cortisolèmie } \\ \square \text { copacité totale de }\end{array}\right.$ liaison

Diagrammes comparatifs de la cortisolémie (F) totale ef de capacité totale de liaison spécifique (NS) au cours de la période néonatale :

FIG. 1. - Chez le cobaye mâle.

FIG. 2. - Chez le cobaye femelle. 
un dimorphisme apparaît en faveur de la femelle qui persistera tou au long de la vie néonatale de l'animal et chez l'adulte et qui est toujours statistiquement significatif $(P<0,001)$.

\section{Discussion et conclusion.}

De l'ensemble des résultats expérimentaux obtenus sur les paramètres étudiés de la fonction surrénalienne en période néonatale, on peut retenir les faits suivants :

- L'évolution de la cortisolémie montre que les surrénales du nouveau-né sont capables de synthétiser el de sécréter, dès la naissance, un taux basal de corticostéroïdes. Cette production est compatible avec les observations histologiques et biochimiques qui attribuent une capacité fonctionnelle à ces glandes dès la vie fœtale aussi bien chez le Cobaye (Dalle et Delost, 1976) que dans d'autres espèces (Alexander ef al., 1968 ; Christianson et Jones, 1957).

- Les cortisolémies très élevées enregistrées en période post-natale (stades $0 \mathrm{~h}$ et $50 \mathrm{~h}$ ) confirment les observations de Malinowska ef al. (1972) et de Cohen (1976) chez le Rat, de Dalle et Delost (1974) chez le Cobaye. Plusieurs hypothèses sont envisageables concernant ce phénomène. L'origine pourrait en être, en partie, maternelle. Chez le Cobaye, Jones (1974) note en fin de gestation une diminution du cortisol libre dans le compartiment maternel et son augmentation dans le compartiment fœtal, suggérant un transfert placentaire de l'hormone de la mère vers le fœtus. Elle pourrait également correspondre à une sécrétion d'origine purement fœtale. Les preuves d'une activité des surrénales fœtales du Cobaye en période prénatale ont été apportées par Dalle et Delost (1976). On sait que l'élévation du cortisol chez le fœtus de Lapin, en fin de gestation, est liée au fonctionnement de l'axe hypophyso-surrénalien de l'embryon (Jost et Jacquot, 1955) ; ce phénomène a également été mis en évidence chez le Rat (Dupouy ef al., 1975).

En outre, le maintien au cours des premiers jours de la vie d'une cortisolémie élevée chez le jeune en opposition avec la chute prononcée ef rapide observée chez la mère en période post partum (Dalle et Delost, 1976) pourrait être associé à la faible capacité du foie à métaboliser les corticostéroïdes sécrétés ou transférés par la mère à la naissance (Shapiro ef al., 1971; Dalle et al., 1980).

- La constante d'association $\mathrm{Ka}$, grandeur moléculaire reflétant la complémentarité de structure existant entre le stéroïde ef le site de fixation de la protéine, ne varie pas aux différents stades étudiés et reste sensiblement égale à $0,25,10^{7} \mathrm{M}^{-1}$ (à $4^{\circ} \mathrm{C}$ ). Cette valeur bien que caractéristique d'interactions spécifiques est relativement faible par rapport à celle d'autres espèces, comme l'Homme (Seal et Doe, 1962) et le Mouton (Fairclough ef Liggins, 1975).

- Les fortes activités liantes spécifiques trouvées à la naissance et dans les premières heures de la vie sont à opposer à la faible liaison des corticostéroïdes dans le compartiment fœtal durant la gestation (Milgrom et al., 1973 ; Savu, 1975). Il faut donc admettre une perméabilité du placenta aux protéines maternelles dans les heures précédant la mise-bas. Cetfe hypothèse s'accorde d'une part avec la capacité de liaison élevée de la CBG dans le compartiment maternel en fin de gestation décrite par Diamond et al. (1969), d'autre part avec la structure du placenta de Cobaye décrite par Kaufmann et 
Davidoff (1977). De type lacunaire, il est caractérisé par de nombreuses anastomoses vasculaires entre les réseaux de la mère et du fotus, anastomoses qui se développent dans les heures précédant la mise-bas. Il y aurait ainsi une situation favorable au passage transplacentaire de la mère vers le fœlus non seulement du cortisol (Dalle ef Delost, 1979), mais également des protéines vectrices qui se retrouveraient ainsi dans la circulation du nouveau-né.

- La mise en parallèle dans les figures 1 et 2 au cours de la période néonatale, de la cortisolémie et de la capacité totale de liaison spécifique du plasma permet de remarquer la simultanéité des valeurs élevées de ces deux paramètres au cours de la première semaine de la vie.

La conséquence en serait le maintien de la fraction libre de l'hormone dans des limites raisonnables. A partir du 10 e jour s'établit aussi bien chez le mâle que chez la femelle la situation rencontrée chez l'animal adulte chez lequel la capacité de liaison CBG devient supérieure à la cortisolémie et peut donc en tamponner les variations éventuelles.

- L'établissement de la capacité liante de la CBG chez le Cobaye en période néonatale présente également une certaine originalité comparativement à d'autres espèces. Chez le Rat (Van Baelen ef al., 1977) et chez l'Homme (Hadjian ef al., 1975), cette période se caractérise par une faible activité liante du plasma sans dimorphisme sexuel perceptible ; le taux de CBG augmente ensuite pour tendre vers les valeurs de l'adulte, atteintes au cours du $2^{\mathrm{e}}$ mois chez le Rat et au bout d'un an seulement chez l'Homme. Nos résultats chez le Cobaye montrent une évolution différente sans passage par un minimum. Les capacités CBG diminuent à partir de la naissance jusqu'à ce qu'elles atteignent leur niveau normal où elles se stabilisent. Celui-ci est obtenu dès la $50^{\mathrm{e}}$ heure chez la femelle, seulement au $20^{\mathrm{e}}$ jour chez le mâle.

- Le dimorphisme sexuel en faveur de la femelle, apparent dès le 7e jour, a peut-être son origine dans les fortes concentrations d'androgènes plasmatiques trouvées chez le mâle au cours de cette période (Pelardy et Delost, 1973), androgènes dont l'effet dépresseur sur l'activité CBG est bien établi (Westphal, 1971).

Il apparaît donc que la période néonatale est chez le Cobaye un moment privilégié au cours duquel l'ajustement des divers paramètres de l'activité surrénalienne concourf à établir dans les meilleurs délais les équilibres caractéristiques de l'état adulte.

Reçu en avril 1980.

Accepté en mars 1981.

\section{Références}

ALEXANDER D., BRITTON H. G., JAMES U., NIXON D. A., PARKER R. A., WINTOUR E., WRIGHT R., 1968. Steroid secretion by the adrenal gland of fetal and neonatal sheep. J. Endocr., 40, $1-13$.

BUSH I. E., 1952. Methods of paper chromatography of steroids applicable to the study of steroids in mammalian blood and tissue. Biochem. Journ., 50, 370-375.

CHRISTIANSON M., JONES T. C., 1957. The interrelationships of the adrenal glands of mother and fetus in the rat. J. Endocr., 15, 17-42.

COHEN A., 1976. Adrenal and plasma corticosterone levels in the pregnant, foetal and neonatal rat in the perinatal period. Horm. Metab. Res., 8, 474-478. 
DALLE M., DELOST P., 1974. Changes in the concentrations of cortisol and corticosterone in the plasma and adrenal glands of the guinea-pig from birth to weaning. $J$. Endocr., 63, 483-488.

DALLE M., DELOST P., 1976. Plasma and adrenal cortisol concentration in foetal, newborn and mother guinea-pig during the perinatal period. J. Endocr., 70, 207-214.

DALLE M., DELOST P., 1979. Foeto-maternal production and transfer of cortisol during the last days of gestation in the guinea-pig. J. Endocr., 82, 43-51.

DALLE M., EL HANI A., DELOOST P., 1980. Changes in cortisol binding and metabolism during neonatal development in the guinea-pig. J. Endocr., 85, 219-227.

DIAMOND M., DEVENUTO F., RUST N., WESTPHAL U., 1969. High affinity binding of progesterone, testosterone and cortisol in normal and androgen treated guinea-pigs during various reproductive stages. Reelationship to masculinization. Endocrinology, 84, 1143-1151.

DUPOUY J. P., COFFIGNY H., MAGRE S., 1975. Maternal and foetal corticosterone levels during late pregnancy in rat. J. Endocr., 65, 347-352.

FAIRCLOUGH R. J., LIGGINS G. C., 1975. Protein binding of plasma cortisol in the foetal lamb near term. J. Endocr., 67, 333-341.

GALA R. R., WESTPHAL U., 1966. Further studies on the corticosteroid-binding globulin in the rat. Proposed endocrine control. Endocrinology, 79, 67-76.

HADJIAN A. J., CHEDIN M., COCHET C., CHAMBAZ E. M., 1975. Cortisol binding to protein in plasma in the human neonate and infant. Ped. Res., 9, 40-45.

JONES C. T., 1974. Corticosteroids concentrations in the plasma of fetal and maternal guinea-pigs during gestation. Endocrinology, 95, 1129-1133.

JOST A., JACQUOT R., 1955. Recherche sur les facteurs endocriniens de la charge en glycogène du foie foetal chez le lapin. Ann. Endocr., Paris, 16, 849-851.

KAUFMANN P., DAVIDOFF M., 1977. The guinea-pig placenta. Springer Verlag, Berlin, Heidelberg, New York, 90 PP.

MALINOWSKA K. W., HARDY R. N., NATHANIELSZ P. W., 1972. Plasma adrenocorticosteroid concentrations immediatly after birth in the rat, rabbit and guinea-pig. Experientia, 28, 13661367.

MILGROM E., ALLOUCH P., ATGER M., BAULIEU E. E., 1973. Progesterone-binding plasma protein of pregnant guinea-pig. J. biol. Chem., 248, 1106-1114.

MURPHY B. E. P., 1967. Some studies of the protein-binding of steroids and their application to the routine micro and ultramicro measurement of various steroids in body fluids by competitive protein-binding radioassay. J. clin. Endocr., 27, 973-990.

PELARDY G., DELOST P., 1973. Evolution des teneurs plasmatiques en testostérone et en androstènedione chez le cobaye mâle au cours des trois premières semaines de la vie. C. R. Acad. Sci. Paris, Sér. D, 277, 949-952.

SAVU L., 1975. Protéines plasmatiques liant les astrogènes ef les glucocorticoïdes au cours du développement fœial ef de la gestation. Eludes comparatives chez le rat, la souris, le cobaye ef l'homme. Th. Doct. Sci., Paris VI, 153 pp.

SCATCHARD G., 1949. The aftractions of proteins for small molecules and ions. Ann. N. Y. Acad. Sci., 51, 660-672.

SEAL U. S., DOE R. P., 1962. Purification and properties of transcortin, the cortisol binding globulin from patients with cancer of the prostate. Cancer Chemother. Rep., 16, 329-331.

SHAPIRO S., PERCIN C. J., KOTICHAS F. J., 1971. Half-life of plasma corticosterone during development. Endocrinology, 89, 284-286.

TOPERT M., ZABEL I., ZIEGLER M., 1974. Thin layer gel filtration as a method for routine steroid receptor binding słudies. Analyt. Biochem., 62, 514-522.

VAN BAELEN H., VAN DOREN G., DE MOOR P., 1977. Concentration of transcortin in the pregnant rat and its foetus. J. Endocr., 75, 427-431.

WESTPHAL U., 1967. Steroid-protein interactions. XIII. Concentrations and binding affinities of corticosteroid-binding globulins in sera of man, monkey, rat, rabbit and guinea-pig. Arch. Biochem., 118, 556-563.

WESTPHAL U., 1971. Steroids protein inferactions. In "Monographs on Endocrinology », Springer Verlag, 567 pp. 